\title{
Dural venous sinus angioplasty and stenting for the treatment of idiopathic intracranial hypertension
}

\author{
Jeremy D Fields, ${ }^{1}$ Parisa P Javedani, ${ }^{2}$ Julie Falardeau, ${ }^{3}$ Gary M Nesbit, ${ }^{1}$ Aclan Dogan, ${ }^{1}$ \\ Erek K Helseth, ${ }^{1}$ Kenneth C Liu, ${ }^{4}$ Stanley L Barnwell, ${ }^{1}$ Bryan D Petersen ${ }^{1}$
}

\begin{abstract}
${ }^{1}$ Department of Interventional Neuroradiology, Oregon Health and Science University, Portland, Oregon, USA ${ }^{2}$ School of Medicine, Oregon Health and Science University, Portland, Oregon, USA ${ }^{3}$ Department of Neuro-ophthalmology, Oregon Health and Science University, Portland, Oregon, USA

${ }^{4}$ Department of Neurosurgery, University of Virginia Health System, Charlottesville, Virginia, USA
\end{abstract}

\section{Correspondence to} Dr Jeremy D Fields, Department of Interventional Neuroradiology, Oregon Health and Science University, 3181 SW Sam Jackson Park Road, CR-127, Portland, OR 97239, USA; fieldsje@ohsu.edu

Received 4 October 2011 Revised 8 November 2011 Accepted 13 November 2011 Published Online First 5 December 2011

\begin{abstract}
Background Lumboperitoneal shunt (LPS),

ventriculoperitoneal shunt (VPS) and optic nerve sheath fenestration (ONSF) are accepted surgical therapies for medically refractory idiopathic intracranial hypertension (IIH). In the subset of patients with $\mathrm{IH}$ and venous sinus stenosis, dural venous sinus stenting has emerged as an alternative surgical approach.

Methods All cases of dural stents for $\mathrm{IH}$ at our institution were retrospectively reviewed. Eligibility criteria included medically refractory $\mathrm{IH}$ with documented papilledema and dural venous sinus stenosis of the dominant venous outflow system (gradient $\geq 10 \mathrm{~mm} \mathrm{Hg}$ ).
\end{abstract}

Results Fifteen cases (all women) of mean age 34 years were identified. All had failed medical therapy and six had failed surgical intervention. Technical success was achieved in all patients without major periprocedural complications. The mean preprocedural gradient across the venous stenosis was reduced from $24 \mathrm{~mm} \mathrm{Hg}$ before the procedure to $4 \mathrm{~mm} \mathrm{Hg}$ after the procedure. Headache resolved or improved in 10 patients. Papilledema resolved in all patients and visual acuity stabilized or improved in 14 patients. There were no instances of restenosis among the 14 patients with follow-up imaging.

Conclusion In this small case series, dural sinus stenting for $\mathrm{IIH}$ was performed safely with a high degree of technical success and with excellent clinical outcomes. These results suggest that angioplasty and stenting for the treatment of medically refractory $\mathrm{IH}$ in patients with dural sinus stenosis warrants further investigation as an alternative to LPS, VPS and ONSF.

\section{INTRODUCTION}

Idiopathic intracranial hypertension (IIH), also known as pseudotumor cerebri and benign intracranial hypertension, is a rare disorder characterized by increased intracranial pressure (ICP) without an intracranial mass. The overall prevalence in North America is approximately $0.9-1.07 / 100000$ but rises to 15-19/100000 among women aged 20-44 years who are overweight ( $\geq 20 \%$ above their ideal bodyweight). ${ }^{1}$ The most widely accepted criteria for diagnosis require: (1) symptoms and (2) signs referable only to elevated intracranial pressure; (3) cerebrospinal fluid (CSF) opening pressure $>25 \mathrm{~cm} \mathrm{H} \mathrm{H}_{2} \mathrm{O}$ measured in the lateral decubitus position; (4) normal CSF composition; and (5) no evidence for an underlying structural cause (using
MRI or contrast-enhanced CT for typical patients and MRI and MR venography for all others). ${ }^{2}$ The most common symptoms are headache, visual disturbance and tinnitus. Papilledema is present in at least $95 \%$. Although headache may be severe, visual loss due to prolonged papilledema and secondary optic atrophy remains the most feared complication and may progress without recognition by the patients themselves. ${ }^{3} 4$

Medical treatment generally consists of a combination of carbonic anhydrase inhibitors such as acetazolamide or topiramate (to decrease CSF production) and symptomatic treatment for headache. For medically refractory patients, two surgical strategies have most commonly been employedCSF diversion with ventriculoperitoneal or lumboperitoneal shunts (VPS or LPS) and optic nerve sheath fenestration (ONSF). While progression of visual loss may be prevented by these surgical approaches, both have significant limitations. Both VPS and LPS have variable clinical response rates and significant shunt failure rates requiring subsequent surgical revision. ${ }^{5}$ Moreover, LPS may lead to a number of adverse events including acquired Chiari syndromes, low pressure headaches and archnoiditis of the lumbosacral nerve roots. ${ }^{6}$ Although ONSF often temporarily arrests visual loss associated with IIH, recurrence of visual symptoms is common and the procedure does not address headache, tinnitus or other symptoms associated with elevated intracranial pressure.

The underlying pathophysiology of IIH has yet to be fully defined, but a number of recent investigations have implicated stenosis of the dural venous sinuses as a potential contributor to the syndrome of IIH in a subset of patients. Transverse sinus stenosis was demonstrated by MRI in $90 \%$ of patients with $\mathrm{IIH}^{7}$ although the degree to which venous sinus stenosis on MRI corresponds to transstenosis pressure gradients is not known. Furthermore, elevation of pressures within the dural sinuses proximal to the stenosis is common and significant pressure gradients across the venous stenoses have been demonstrated by direct manometry in patients with IIH. $^{8} 9$ Although considerable controversy exists regarding whether dural venous stenosis is a cause of IIH or an effect of elevated intracranial pressure, the results of several case series suggest that stenting of the stenotic dural venous sinus may represent a therapeutic option for medically refractory IIH. ${ }^{3} 1011$ In this paper we present our single-center experience with dural venous sinus stenting for IIH. 


\section{METHODS}

We retrospectively reviewed all cases of patients treated with dural stents for IIH with papilledema at our institution. The study protocol was approved by our institutional review board.

\section{Patient selection and characteristics}

Patients were considered eligible for stenting based on the following criteria: (1) medically refractory IIH; (2) papilledema confirmed by an ophthalmologist; and (3) dural venous sinus stenosis of the dominant venous outflow system with a gradient of $\geq 10 \mathrm{~mm} \mathrm{Hg}$. This cut-off pressure was selected because a gradient of $5-6 \mathrm{~mm} \mathrm{Hg}$ from the superior sagittal sinus to the internal jugular bulb is present in normal controls. ${ }^{9}$

Demographic features (age, gender, body mass index (BMI)), prior treatments (medical and surgical), CSF opening pressures, neuro-ophthalmologic evaluation, procedural details and clinical outcomes were obtained by retrospective chart review. Headache intensity and the presence of tinnitus at last follow-up were assessed by a combination of chart review and telephone interview. Headache was evaluated by structured interview and was categorized as: (1) resolved (no further headache); (2) improved (residual symptoms not requiring continued intervention); (3) no change (no change in symptoms compared with before stenting procedure); (4) worsened (worse than before stenting procedure); and (5) different (change of symptoms compared with prior to the stenting procedure not otherwise meeting the criteria for categories 1-4).

\section{Procedure}

Prior to intervention, diagnostic angiography and venous pressure measurements were performed under moderate sedation. A full evaluation of the dural sinuses was performed by study of both internal carotid arteries and the posterior circulation. A $6 \mathrm{~F}$ shuttle sheath (Cook Medical, Bloomington, Indiana, USA) was then placed in the dominant internal jugular vein. A 0.027 inch Renegade microcatheter (Stryker, Kalamazoo, Michigan, USA) was placed coaxially within the 6F shuttle and navigated over a 0.014 inch guidewire into the torcula. The microcatheter was then pulled back from the torcula to the internal jugular vein and simultaneous pressure measurements were obtained from the $6 \mathrm{~F}$ sheath and microcatheter tip. These pressures were recorded to obtain pressure gradients from the torcula, transverse sinus and sigmoid sinus with respect to the internal jugular vein. After identifying a venous sinus stenosis, dural sinus venography was performed through the 0.027 inch microcatheter to define the anatomy of the stenosis and the size of the native dural sinus.

Patients with a dural venous sinus stenosis of the dominant venous outflow system with a gradient of $\geq 10 \mathrm{~mm} \mathrm{Hg}$ were referred for stent placement.

All patients were pretreated with aspirin $325 \mathrm{mg}$ and clopidogrel $75 \mathrm{mg}$ for 5 days or loaded with aspirin $325 \mathrm{mg}$ and clopidogrel $600 \mathrm{mg}$ at least $4 \mathrm{~h}$ prior to the procedure. Intravenous heparin was given during the stent procedure to raise the activated clotting time to $>250 \mathrm{~s}$. Dual antiplatelet therapy was continued for 6 months and aspirin indefinitely thereafter.

All dural sinus stent procedures were performed with the patients under general anesthesia.

First, venous access was achieved in the jugular vein ipsilateral to the intended treatment site via the transfemoral approach. The choice of venous access sheath systems evolved as we gained experience with the procedure in order to provide adequate support for stent placement. The final venous access sheath system was a triaxial construct consisting of 12F, 9F and
7F 80-90 cm length sheaths. This system provided sufficient support for the delivery of the stent to the stenotic region of the transverse sinus.

After placement of the venous sheaths, diagnostic angiography was performed from the ipsilateral internal carotid artery and the venous phase of this study was used as a road map for traversal and stenting of the dural sinuses. Introduced through the venous sheaths, an 0.027 inch Renegade microcatheter was again navigated across the area of stenosis and placed with the distal tip at the level of the torcula. Dual venous pressure measurements were then repeated to confirm the presence of a persistent trans-stenosis gradient. Through this microcatheter, a stiff 0.018 inch guidewire (Platinum Plus; Stryker) exchange length guidewire was placed with the distal tip into the superior sagittal sinus or contralateral transverse sinus. Over this guidewire, a Sterling $6 \mathrm{~mm} \times 40 \mathrm{~mm}$ over-the-wire balloon angioplasty catheter (Stryker) was positioned across the stenosis and then inflated fully at 8-10 atmospheres for $10 \mathrm{~s}$ for two inflations. Following balloon angioplasty, in most cases a Zilver $51810 \mathrm{~mm}$ diameter self-expanding stent (Cook Medical) was positioned across the residual area of narrowing and deployed. The selfexpanding stents ranged from 40 to $80 \mathrm{~mm}$ in length and were chosen to completely cover the stenosis with at least $10 \mathrm{~mm}$ of coverage of the adjacent non-stenotic portion of the vein on each side. A single stent was used to treat the stenotic portion of the vessel in all but one case (for which two stents were used due to inadequate coverage of the stenosis). After initial experience suggested that bilateral stenting was not necessary, unilateral stenting procedures were performed.

Following stent placement, the 0.027 inch lumen microcatheter was then advanced through the stent to the level of the torcula and simultaneous pressure measurements were obtained to verify efficacy of the stenting procedure and elimination of the trans-stenosis pressure gradient. The arteriotomy was then closed with a StarClose device (Abbott, Chicago, Illinois, USA) and the venotomy was closed with manual pressure.

\section{Angiographic follow-up and outcome assessment}

Papilledema and visual assessment by an ophthalmologist was undertaken in all patients before stent placement with initial follow-up examination generally 6 weeks after intervention. Evaluations included visual acuity testing, color vision, automated perimetry (Humphrey or Octopus) and funduscopic evaluation. Angiography to evaluate stent patency and restenosis was performed at 6 months after intervention in most patients, although non-invasive imaging was used in some cases. Clinical assessment took place at the time of follow-up angiography as well on an ad hoc basis if additional concerns arose.

\section{RESULTS}

A total of 15 patients (all women) of mean age 34 years (range 20-56) and mean BMI $39 \mathrm{~kg} / \mathrm{m}^{2}$ pre-procedure and $39 \mathrm{~kg} / \mathrm{m}^{2}$ post-procedure (range $30-73 \mathrm{~kg} / \mathrm{m}^{2}$ pre-procedure and $30-49 \mathrm{~kg} / \mathrm{m}^{2}$ post-procedure) were treated (table 1). Only one patient had a reduction in BMI of $>5 \%$ during the follow-up period (from $73 \mathrm{~kg} / \mathrm{m}^{2}$ to $47 \mathrm{~kg} / \mathrm{m}^{2}$ ). Papilledema (diagnosed by an ophthalmologist) and intractable headache were present in all patients prior to the intervention. Pulsatile tinnitus was present in 14 of the 15 patients (93\%).

All had failed medical treatment with acetazolamide; in addition, topiramate and amitriptyline for the treatment of headache were each administered to three patients $(20 \%)$ and furosemide to two (13\%). Surgical interventions prior to 


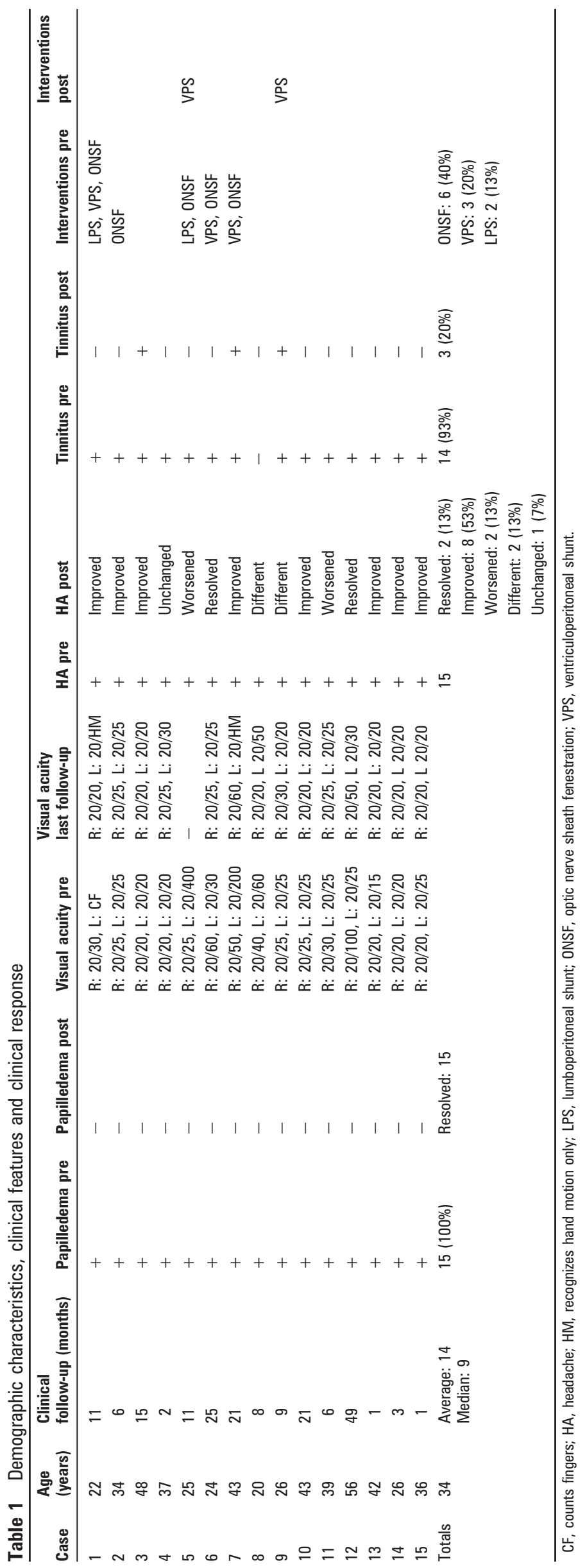




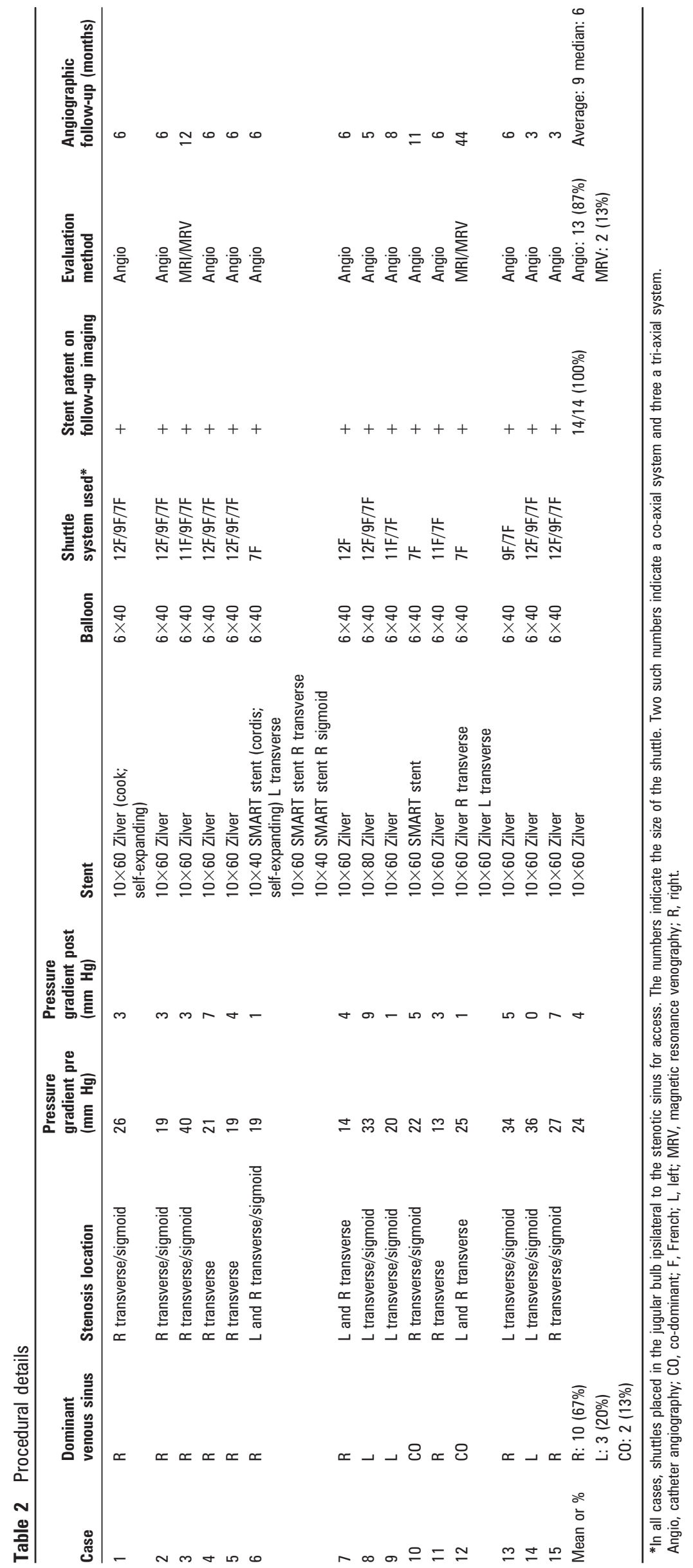


stenting had been performed in six of the patients (40\%): CSF diversion (VPS, LPS or both) in four patients (27\%) and ONSF in $\operatorname{six}(40 \%)$.

Technical success was achieved in all patients with no major complications. One minor complication (7\%), a femoral pseudoaneurysm, was successfully treated with ultrasound-guided compression. The procedural details are shown in table 2 and an illustrative case is depicted in figure 1 . The gradient across the stenotic dural sinus was reduced from a mean of $24 \mathrm{~mm} \mathrm{Hg}$ (range 13-40) to $4 \mathrm{~mm} \mathrm{Hg}$ (range 1-7). A $6 \mathrm{~mm} \times 40 \mathrm{~mm}$ balloon was used for pre-stent angioplasty in all patients. In most cases, a $10 \mathrm{~mm} \times 60 \mathrm{~mm}$ self-expanding stent was placed. Stenting was unilateral in 13 of the 15 patients (87\%) and bilateral in two (13\%).

Clinical follow-up was available in all patients at a mean of 14 months (median 9 months) after stenting. Angiographic follow-up was performed in all patients (catheter angiography in 13 and MRV in 2) at a mean of 9 months (median 6 months) after stenting; the remaining patient has yet to undergo her 6month angiogram. Stents were patent without evidence of restenosis. All patients were preloaded with aspirin and clopidogrel and these medications were continued as directed (clopidogrel for 6 months and aspirin indefinitely) in 14 patients; one patient discontinued both antiplatelet agents at 4 months without any untoward clinical effect.
Papilledema resolved in all 15 patients (see figure 2 for representative funduscopic images). Headache resolved or improved in $67 \%$ and tinnitus was eliminated in $79 \%$ of patients reporting tinnitus prior to the intervention. Visual acuity was assessed formally by an ophthalmologist in 14 of the 15 patients. Of the 14 patients with formal visual acuity testing, 13 (93\%) stabilized or improved and one $(7 \%)$ worsened. The patient who worsened progressed from $20 / 200$ vision in the left eye to ability to detect hand motion only; because papilledema had resolved, the presumed mechanism was progression of optic atrophy due to injury sustained prior to stenting. Due to persistent refractory headache, two patients (13\%) underwent VPS following the stenting procedure.

\section{DISCUSSION}

For medically refractory patients with IIH, particularly those with visual loss and intractable headache, surgical options may be considered but, unfortunately, the available surgical interventions for IIH (ie, VPS, LPS and ONSF) have significant limitations. Response rates to CSF diversion vary considerably depending on the series. Stabilization or improvement in vision has been reported to occur in as few as $62 \%$ of patients undergoing VPS or LPS and headaches to improve or resolve in as few as $45 \%$, with frequent recurrences over time. Shunt revisions are necessary in $38-63 \%$ of patients. Furthermore, although rare,
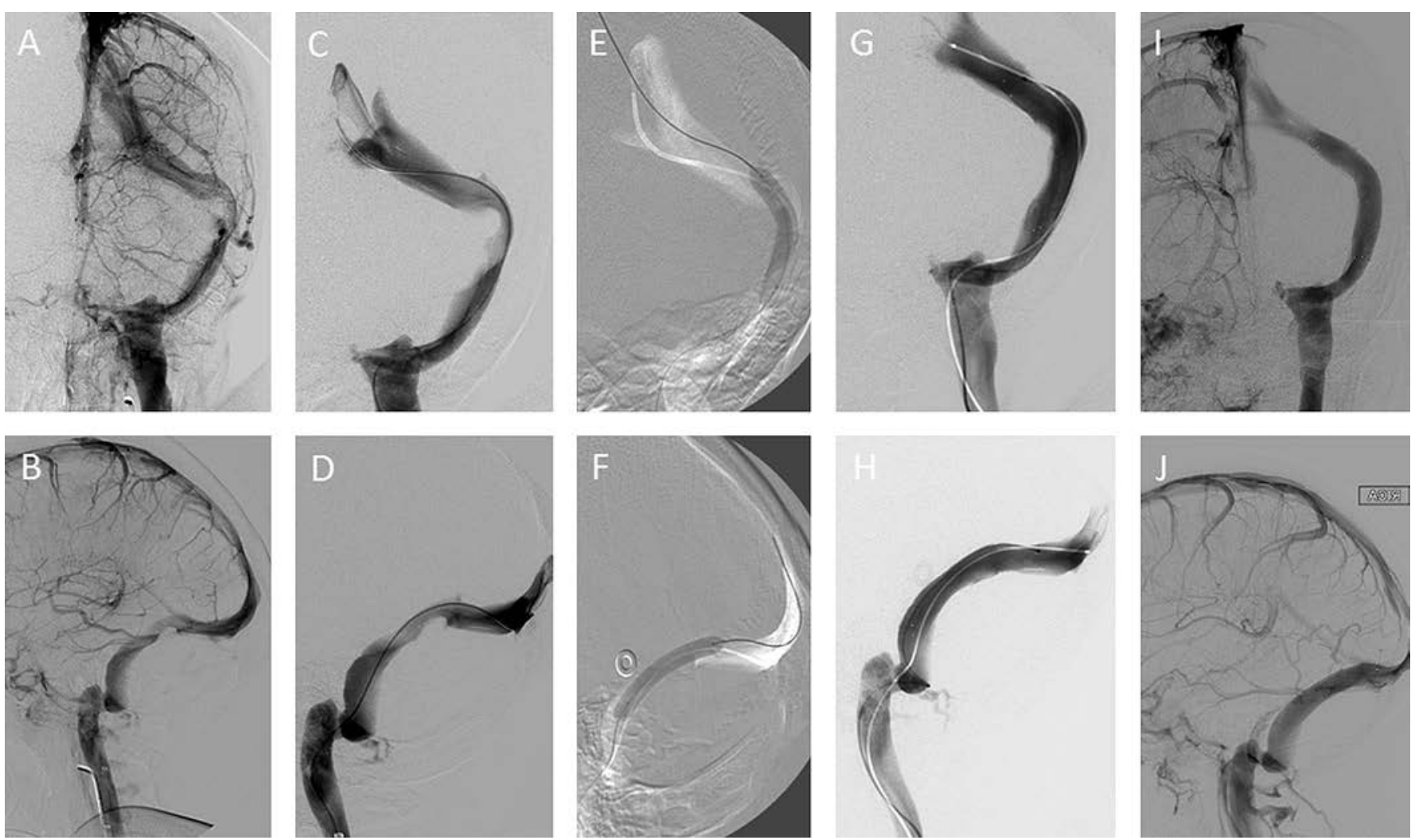

Figure 1 Illustrative case of a 26-year-old woman with idiopathic intracranial hypertension (IIH). (A, B) Left internal carotid artery injection, venous phase, anteroposterior and lateral views. The left transverse sinus system is dominant and a venous stenosis at the transverse-sigmoid sinus junction is demonstrated. (C, D) Microcatheter injection at the torcula, anteroposterior and lateral views, again demonstrating the venous stenosis at the transverse-sigmoid sinus junction. (E, F) Roadmap images, anteroposterior and lateral views, demonstrating pre-stent angioplasty with a $6 \times 40 \mathrm{~mm}$ over-the-wire balloon. The stiff 0.18 inch wire is positioned in the superior sagittal sinus. $(G, H)$ Microcatheter injection at the torcula, anteroposterior and lateral views, immediately after angioplasty followed by deployment of a $10 \times 60 \mathrm{~mm}$ self-expanding stent extending from the lateral aspect of the left transverse sinus to the left sigmoid sinus across the region of stenosis. The left transverse sinus system is now widely patent with evidence of residual stenosis. (I, J) Follow-up angiography 8 months after stent placement. The right internal carotid artery injection, anteroposterior and lateral views, shows no evidence of restenosis. 
Figure 2 Funduscopic examination (A) prior to stenting showing optic disc edema with obscuration of the optic disc margins, elevation of the optic disc and tortuosity of retinal veins and $(B)$ 3 weeks after stenting showing resolution of optic disc edema and improved vascular tortuosity.

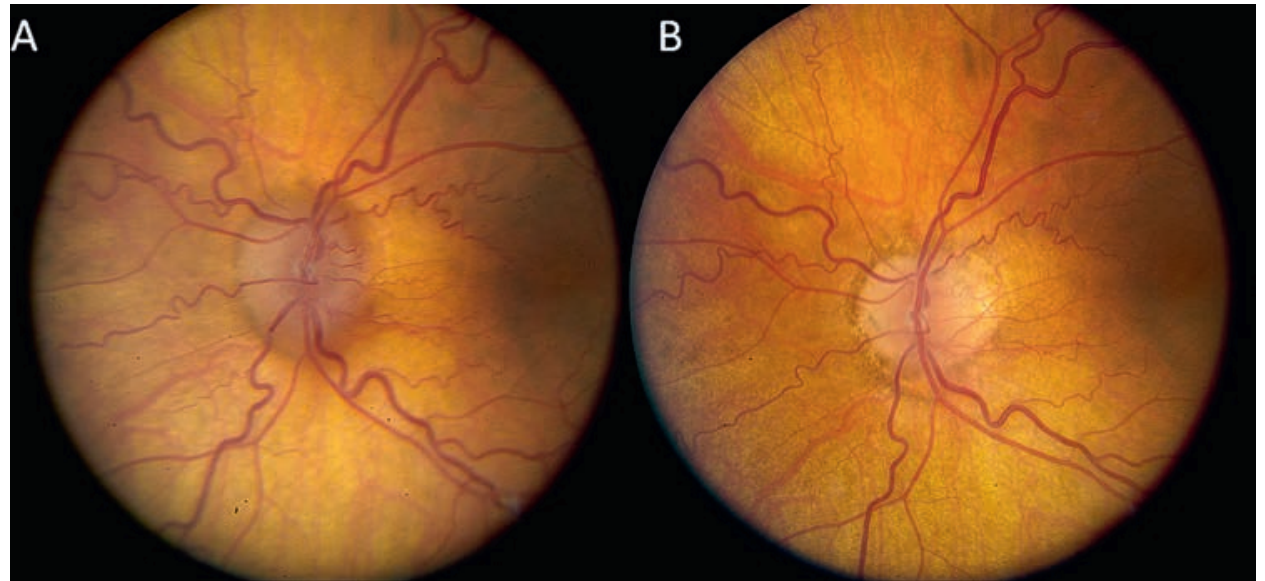

serious complications (including a $0.5 \%$ risk of in-hospital death) have been reported. ${ }^{12}$ Similarly, ONSF is associated with substantial treatment failure rates (worsening in vision after a period of stabilization in $34 \%$ of patients at 1 year and $45 \%$ at 3 years) and failure to improve headache in one-third to one-half. ${ }^{12}$

Given the problems inherent in the traditional surgical treatments for IIH, our results suggest that venous sinus stenting may be a reasonable alternative treatment in the subset of patients with venous sinus stenosis associated with a significant pressure gradient and, indeed, may treat the underlying etiology of the disease. Of the 15 patients treated, six had previously failed treatment with LPS, VPS or ONSF, including two patients who had undergone all three treatments. Visual acuity stabilized or improved in $93 \%$ of patients and papilledema resolved in all patients treated. Headaches improved or resolved in $67 \%$. On follow-up imaging, all stents remained patent with no instances of in-stent thrombosis or restenosis.

Our results are consistent with those reported in previous case series. A systematic review of all published case series was recently performed, encompassing a total of 40 patients (37 undergoing unilateral stents and three bilateral). Among the 40 patients described in these studies, headache resolved or improved in 33 (83\%), papilledema resolved or improved in 30 $(75 \%)$ and optic atrophy occurred in eight $(20 \%) .{ }^{11}$ Since the publication of this review, two relatively large single-center case series have been reported. In one series of 18 patients (with clinical follow-up in 15), $80 \%$ of patients had improvement or resolution of headache after stenting. ${ }^{13}$ The results of ophthalmologic examination were not reported in this study. Most recently, in a series of 52 patients, papilledema was eliminated in all patients and headache in 44. Among the eight patients without resolution of headache, six were found to have restenosis adjacent to the stent, resulting in an overall rate of restenosis of $12 \%{ }^{14}$

Several technical considerations contribute to successful stenting and to symptom resolution. Venous sinuses require relatively large stents; we used $10 \mathrm{~mm}$ self-expanding stents in most cases and did not observe any instances of in-stent stenosis or restenosis of the adjacent dural sinus. The use of stents $10 \mathrm{~mm}$ in diameter may account for the absence of restenosis or stenosis of the adjacent dura observed in our study compared with the $12 \%$ rate of restenosis seen in the aforementioned case series. Due to the tortuosity inherent in the sigmoid sinus/ transverse sinus system and the stiffness of the stent, a robust support system is required for adequate access. After evaluating several different configurations, we found a triaxial 12F/9F/7F shuttle system placed within the internal jugular vein provided adequate support to ensure stent delivery. Moderate oversizing of the stent diameter with respect to the dural sinus assures adequate apposition of the stent to the venous wall, theoretically decreasing clot formation and increasing patency. In addition, short-term restenosis may result from inadequate coverage or progression of the lesion and therefore relatively long stents were employed. $^{15}$

The pathophysiologic relationship between IIH and venous sinus stenosis remains controversial. Undoubtedly, dural venous sinus stenosis is much more common in patients with IIH than controls. ${ }^{716}$ Nonetheless, it remains unclear whether elevations in intracranial pressure are responsible for transverse sinus stenosis or transverse sinus stenosis causes elevations in intracranial pressure. ${ }^{16}$ Regardless, the high response rates to transverse sinus stenting seen in our patients as well as those from previous investigations strongly suggests that preserving the patency of the stenotic transverse sinus frequently ameliorates the pathophysiologic mechanism that ultimately leads to IIH, either by addressing the underlying venous stenosis or by preventing venous sinus collapse in the face of elevated intracranial pressure

This study has a number of limitations. Invasive procedures are frequently associated with a high placebo response rate and the clinical course of IIH inherently fluctuates. Therefore, in the absence of a control group, the overall improvement in subjective symptoms such as headache and tinnitus could result from a placebo effect or the natural history of the disease rather than from the stenting procedure per se. On the other hand, papilledema was evaluated by ophthalmologists and was found to have resolved in all patients-an objective finding unlikely to result from either placebo or natural history.

\section{CONCLUSIONS}

We retrospectively reviewed the results of venous sinus stenting for IIH in 15 consecutive patients treated at a single institution. Patients were selected if they had IIH with papilledema and a venous sinus stenosis with a gradient $\geq 10 \mathrm{~mm} \mathrm{Hg}$ across the stenosis. Technical success and resolution of papilledema was achieved in all patients without any significant procedural complications. Headache improved or completely resolved in $67 \%$. These results provide additional support for venous stenting as a surgical alternative in a properly selected subset of patients with IIH. Moreover, the high rate of clinical improvement following stenting provides additional evidence for the 


\section{Key messages}

Traditional surgical treatments for medically refractory idiopathic intracranial hypertension (IIH) - ventriculoperitoneal shunting (VPS), lumboperitoneal shunting (LPS) and optic nerve sheath fenestration (ONSF) - are associated with significant rates of complications and lack of clinical response. Stenting of the transverse sinus in patients with $\mathrm{IH}$ and transverse sinus stenosis of the dominant cerebral venous sinus has been proposed as a potential alternative therapy.

- We retrospectively reviewed the procedural details and outcomes of stenting for the treatment of medically refractory IIH at our institution.

- Thirteen patients were treated with transverse sinus stenting with a technical success rate of $100 \%$, improvement or resolution of headache in $69 \%$, resolution or improvement of papilledema in all patients and stabilization or improvement of visual acuity in $92 \%$.

- These results suggest that venous sinus stenting in appropriately selected patients with medically refractory IIH may be a reasonable alternative to VPS, LPS and ONSF.

hypothesis that dural venous sinus stenosis contributes to the underlying etiology of IIH in a subset of patients.

\section{Competing interests None.}

Patient consent Because this is a retrospective study with information derived from retrospective chart review, no consent was necessary from the patients. All data were deidentified/anonymized and acquired and stored in a manner designed to protect patient confidentiality in accordance with the policies of the IRB.

Ethics approval Ethics approval was obtained from the Oregon Health and Science University Institutional Review Board.
Contributors All authors contributed to the conception, design, editing and authorship of this study. JDF and PPJ contributed equally to the preparation of this manuscript.

Provenance and peer review Not commissioned; externally peer reviewed.

\section{REFERENCES}

1. Durcan FJ, Corbett JJ, Wall M. The incidence of pseudotumor cerebri. Population studies in lowa and Louisiana. Arch Neurol 1988:45:875-7.

2. Friedman DI, Jacobson DM. Diagnostic criteria for idiopathic intracranial hypertension. Neurology 2002:59:1492-5.

3. Donnet A, Metellus P, Levrier 0, et al. Endovascular treatment of idiopathic intracranial hypertension: clinical and radiologic outcome of 10 consecutive patients. Neurology 2008;70:641-7.

4. Randhawa S, Van Stavern GP. Idiopathic intracranial hypertension (pseudotumor cerebri). Curr Opin Ophthalmol 2008;19:445-53.

5. Brazis PW. Clinical review: the surgical treatment of idiopathic pseudotumour cerebri (idiopathic intracranial hypertension). Cephalalgia 2008;28:1361-73.

6. Ball AK, Clarke CE. Idiopathic intracranial hypertension. Lancet Neurol 2006;5:433-42.

7. Farb RI, Vanek I, Scott JN, et al. Idiopathic intracranial hypertension: the prevalence and morphology of sinovenous stenosis. Neurology 2003;60:1418-24.

8. Karahalios DG, Rekate HL, Khayata $\mathrm{MH}$, et al. Elevated intracranial venous pressure as a universal mechanism in pseudotumor cerebri of varying etiologies. Neurology 1996;46:198-202.

9. King J0, Mitchell PJ, Thomson KR, et al. Cerebral venography and manometry in idiopathic intracranial hypertension. Neurology 1995;45:2224-8.

10. Higgins JN, Cousins C, Owler BK, et al. Idiopathic intracranial hypertension: 12 cases treated by venous sinus stenting. J Neurol Neurosurg Psychiatry 2003:74:1662-6.

11. Bussiere M, Falero R, Nicolle D, et al. Unilateral transverse sinus stenting of patients with idiopathic intracranial hypertension. AJNR Am J Neuroradiol 2010:31:645-50.

12. Uretsky S. Surgical interventions for idiopathic intracranial hypertension. Curr Opin Ophthalmol 2009;20:451-5.

13. Albuquerque FC, Dashti SR, Hu YC et al. Intracranial venous sinus stenting for benign intracranial hypertension: clinical indications, technique, and preliminary results. World Neurosurg 2011;75:648-52; discussion 592-5.

14. Ahmed RM, Wilkinson M, Parker GD, et al. Transverse sinus stenting for idiopathic intracranial hypertension: a review of 52 patients and of model predictions. AJNR Am J Neuroradiol 2011;32:1408-14.

15. Tsumoto T, Miyamoto T, Shimizu M, et al. Restenosis of the sigmoid sinus after stenting for treatment of intracranial venous hypertension: case report. Neuroradiology 2003:45:911-15

16. Friedman DI. Cerebral venous pressure, intra-abdominal pressure, and dural venous sinus stenting in idiopathic intracranial hypertension. J Neuroophthalmol 2006;26:61-4 